The impact of sharp screening on the Coulomb scattering problem in three dimensions

This article has been downloaded from IOPscience. Please scroll down to see the full text article.

2010 J. Phys. A: Math. Theor. 43245302

(http://iopscience.iop.org/1751-8121/43/24/245302)

View the table of contents for this issue, or go to the journal homepage for more

Download details:

IP Address: 92.42.26.210

The article was downloaded on 19/05/2010 at 13:05

Please note that terms and conditions apply. 


\title{
The impact of sharp screening on the Coulomb scattering problem in three dimensions
}

\author{
S L Yakovlev ${ }^{1}$, M V Volkov ${ }^{2,3}$, E Yarevsky $^{1}$ and N Elander ${ }^{3}$ \\ ${ }^{1}$ Department of Computational Physics, St Petersburg State University, 198504 St Petersburg, \\ Russia \\ ${ }^{2}$ Department of Quantum Mechanics, St Petersburg State University, 198504 St Petersburg, \\ Russia \\ ${ }^{3}$ Department of Physics, Stockholm University, Alba Nova University Center, SE 10691 , \\ Stockholm, Sweden \\ E-mail: yakovlev@cph10.phys.spbu.ru
}

Received 1 March 2010

Published 19 May 2010

Online at stacks.iop.org/JPhysA/43/245302

\begin{abstract}
The scattering problem for two particles interacting via the Coulomb potential is examined for the case where the potential has a sharp cut-off at some distance. The problem is solved for two complementary situations, firstly, when the interior part of the Coulomb potential is left in the Hamiltonian and, secondly, when the long-range tail is considered as the potential. The partial wave results are summed up to obtain the wavefunction in three dimensions. It is shown that in the domains where the wavefunction is expected to be proportional to the known solutions, the proportionality is given by an operator acting on the angular part of the wavefunction. The explicit representation for this operator is obtained in the basis of Legendre polynomials. We proposed a driven Schrödinger equation including an inhomogeneous term of the finite range with purely outgoing asymptotics for its solution in the case of the three-dimensional scattering problem with long-range potentials.
\end{abstract}

PACS number: 03.65.Nk

\section{Introduction}

\subsection{Background}

The Coulomb force is the dominating interaction in atomic and molecular physics. It is therefore the underlying force in chemistry and biology. Describing reactions with charged particles is thus an essential task for theoretical atomic and molecular physics as well as in chemistry. Despite this fundamental importance, solving the Schrödinger equation for even 
a three-body problem is a very difficult task. The asymptotic form of the wavefunction for three-body breakup is known to have a very complicated structure. A recent review [1] provided an outline of the most important articles which deal with the scattering problem of charged particles. The majority of these methods focus on solving the three-body problem without explicit knowledge of the three-body breakup asymptotics. Inspired by the methods of [2] and [3, 4], in two recent studies we presented a new and rigorous method which can be used to solve the Coulomb scattering problem by using exterior complex scaling $[5,6]$. The former analyzes the single-channel two-body problem, while the latter indicates how the same formalism can be generalized to the full multi-channel three-body problem. In this contribution, we demonstrate the construction of the formal part of the three-dimensional formulation of the three-body problem outlined in [6] by studying the three-dimensional two-body problem.

\subsection{Definition of the problem}

The scattering solution to the Schrödinger equation

$$
\left[-\Delta_{\mathbf{r}}+V_{\mathrm{c}}(r)-k^{2}\right] \psi_{\mathrm{c}}(\mathbf{r}, \mathbf{k})=0
$$

for the Coulomb potential $V_{\mathrm{c}}(r)=2 \eta k / r$ has the well-known form [7-9]

$$
\psi_{\mathrm{c}}(\mathbf{r}, \mathbf{k})=\Gamma(1+\mathrm{i} \eta) \mathrm{e}^{-\pi \eta / 2} \mathrm{e}^{\mathrm{ir} \cdot \mathbf{k}}{ }_{1} F_{1}(-\mathrm{i} \eta, 1, \mathrm{i}(r k-\mathbf{r} \cdot \mathbf{k})) .
$$

Here the vectors $\mathbf{r}$ and $\mathbf{k}$ describe the position and the incident momentum. Their magnitudes are denoted by $r$ and $k$. $\Gamma$ and ${ }_{1} F_{1}$ are the gamma function and the confluent hypergeometric function, respectively. Another way of representing the solution $\psi_{\mathrm{c}}(\mathbf{r}, \mathbf{k})$ is the partial wave decomposition [9]:

$$
\psi_{\mathrm{c}}(\mathbf{r}, \mathbf{k})=\frac{1}{k r} \sum_{\ell=0}^{\infty}(2 \ell+1) \mathrm{i}^{\ell} \mathrm{e}^{\mathrm{i} \sigma_{\ell}} F_{\ell}(\eta, k r) P_{\ell}(\cos \theta) .
$$

Here $\cos \theta=\mathbf{r} \cdot \mathbf{k} / r k, \sigma_{\ell}=\arg \Gamma(\ell+1+\mathrm{i} \eta)$ represents the Coulomb phase shift and $P_{\ell}$ is the $\ell$ th Legendre polynomial. The regular Coulomb wavefunction $F_{\ell}(\eta, k r)$ [10] obeys the partial wave equation

$$
\left(-\frac{\mathrm{d}^{2}}{\mathrm{~d} r^{2}}+\frac{\ell(\ell+1)}{r^{2}}+\frac{2 \eta k}{r}-k^{2}\right) F_{\ell}(\eta, k r)=0
$$

and the boundary condition $F_{\ell}(\eta, 0)=0$ at the origin. With this choice of coordinates, the wavefunction depends on the triad $r, u=\cos \theta, k$, such that $\psi_{\mathrm{c}}=\psi_{\mathrm{c}}(r, u, k)$.

Although the explicit representations (2) and (3) have been known since the earliest stages of quantum mechanics $[7,8]$, the various approximations, which are based on the procedures necessary for the suppression of the long-range tail of the Coulomb potential, have been studied for many decades. The total number of publications on this subject is enormous, and so here we quote only those few which focus on the principal aspects of the problem [7, 8, 11-13]. Such procedures, called screening, are of substantial interest in view of their application to the scattering problem for more than two particles, since in that case the exact solution of the Coulomb problem is not available $[14,15]$.

In principal, two kinds of screening procedures exist, i.e. the sharp and the soft. The sharp procedure, which cuts off the Coulomb potential beyond some radius $R$, leads to the finite-range potential

$$
V_{R}(r)=V_{\mathrm{c}}(r) \Theta(R-r)
$$


Here $\Theta$ is the standard Heaviside function such that $\Theta(t)=1(0), t \geqslant 0(t<0)$. Soft screening methods imply multiplication of $V_{\mathrm{c}}$ by a smooth screening factor as, for instance, is done in the Yukawa potential $W_{\rho}(r)=V_{\mathrm{c}}(r) \mathrm{e}^{-r / \rho}$. The Coulomb wavefunction can be obtained in the limit $\rho \rightarrow \infty$ from the solutions of the Schrödinger equation with the potential $W_{\rho}$. A regularizing factor is needed to obtain the correct limit. For the three-dimensional Yukawa potential, this factor is known analytically [13].

The situation with the potential $V_{R}$ is more delicate. The representation for the Coulomb wavefunction through the solution for the screened potential $V_{R}$ can easily be obtained at finite values of $R$ for partial wave components [8, 11, 12]. The regularizing factor in this case is $\ell$-dependent. The infinite sum over $\ell$ should be computed to obtain the solution in three dimensions. An accurate analysis of the asymptotics of the partial wave series for the scattering amplitude as $R \rightarrow \infty$ was performed in [12] on the basis of distribution theory. This led the authors to the commonly accepted asymptotic regularizing factor $\mathrm{e}^{-\mathrm{i} 2 \eta \log 2 k R}$ for the scattering amplitude for the potential $V_{R}$. No extra term possessing singularities in the forward (backward) scattering direction was observed in [12], since the test functions used for the partial series summations were assumed to be vanishing in those directions. In fact, this requirement is not necessary. The problem of deriving the correct three-dimensional expression for the wavefunction, which explicitly includes the Coulomb wavefunction $\psi_{\mathrm{c}}$, was not the focus of the papers $[11,12]$. The paper [16] attempted to solve the problem in three dimensions directly by solving the three-dimensional Lippmann-Schwinger equation with the potential $V_{R}$. However, the derivations made in [16] have been performed only for a particular value of the coordinate $r=0$. It was not proven that the solution obtained in [16] is valid for all values of $r$. In the comment [17] it was shown by direct calculations that the three-dimensional result of [16] is erroneous. A further discussion of the results of [16] and their relation to the results of [12] can be found in [18].

To date, the open situation of the cut-off Coulomb problem coupled with our own interests [5], related to the application of the complex rotation method for calculating the scattering states in the system of particles with long-range interactions, stimulated this research. This paper is devoted to studying the scattering problem for a sharp cut-off Coulomb potential $V_{R}$ and its complement

$$
V^{R}=V_{\mathrm{c}}-V_{R}
$$

In section 2, the partial wave equations are solved for a potential $V_{R}$ by the conventional matching procedure at the point $r=R$, and the infinite sum over angular momenta $\ell$ is then evaluated in order to obtain the solution to the three-dimensional Schrödinger equation. The main result of this paper is that we prove in the region $r<R$ the wavefunction for the potential $V_{R}$ in three dimensions, given by the action of an operator on the Coulomb wavefunction. This operator acts over the angular coordinate. The asymptotics of this operator is evaluated as $R \rightarrow \infty$. Our derivation supports the form of the regularizing factor for the wavefunction which was proposed in [11] for three dimensions but without a detailed proof. An extra term is found in the asymptotics of the scattering amplitude which possesses fast oscillations as a function of $R$ and delta-functional singularity in the forward scattering direction. In section 3 , we present the solution of the scattering problem for the Schrödinger equation with the potential $V^{R}$. To the best of the authors' knowledge, this is the first time that this has been reported. This solution is used in section 4 to construct the three-dimensional driven Schrödinger equation with the finite-range potential $V_{R}$ in the inhomogeneous term. As we demonstrated in our recent paper [5], this equation is ideally suitable for applying the complex rotation method to solve the scattering problem with long-range interactions. 


\section{The scattering problem for the potential $V_{R}$}

The partial wave equation

$$
\left(-\frac{\mathrm{d}^{2}}{\mathrm{~d} r^{2}}+\frac{\ell(\ell+1)}{r^{2}}+V_{R}(r)-k^{2}\right) v_{\ell}(r, k)=0
$$

with the boundary condition $v_{\ell}(0, k)=0$ and the asymptotics as $r \rightarrow \infty$

$$
v_{\ell}(r, k) \sim \hat{j}_{\ell}(k r)+A_{R \ell} \hat{h}_{\ell}^{+}(k r)
$$

determines the scattering partial wavefunction for a given orbital momentum $\ell$. Here $\hat{j}_{\ell}$ and $\hat{h}_{\ell}^{+}$ are the standard Riccati-Bessel and Riccati-Hankel functions [10]. The exact representation for $v_{\ell}(r, k)$ has a different form depending on whether the value of $r$ is in or out the interval $0<r \leqslant R$ [11]. For $r \in(0, R]$, one obtains

$$
v_{\ell}(r, k)=a_{R \ell} F_{\ell}(\eta, k r) .
$$

For $r \geqslant R$, the solution $v_{\ell}$ takes the form

$$
v_{\ell}(r, k)=\hat{j}_{\ell}(k r)+A_{R \ell} \hat{h}_{\ell}^{+}(k r) .
$$

At $r=R$, both the function $v_{\ell}$ and its first derivative have to be continuous in $r$, i.e. $\partial_{r}^{n} v_{\ell}(R-0, k)=\partial_{r}^{n} v_{\ell}(R+0, k), n=0,1$. These conditions yield

$$
a_{R \ell}=W_{R}\left\{\hat{j}_{\ell}, \hat{h}_{\ell}^{+}\right\} / W_{R}\left\{F_{\ell}, \hat{h}_{\ell}^{+}\right\}
$$

and

$$
A_{R \ell}=W_{R}\left\{\hat{j}_{\ell}, F_{\ell}\right\} / W_{R}\left\{F_{\ell}, \hat{h}_{\ell}^{+}\right\},
$$

where $W_{R}\{f, g\}$ is the Wronskian $f(r) g^{\prime}(r)-f^{\prime}(r) g(r)$ that is calculated at $r=R$. The phase shift $\delta_{R \ell}$ is then determined by the standard representation of the scattering amplitude $A_{R \ell}$ :

$$
A_{R \ell}=\frac{\mathrm{e}^{\mathrm{i} 2 \delta_{R \ell}}-1}{2 \mathrm{i}} .
$$

It is seen from (11) and (12) that the phase shift $\delta_{R \ell}$ can also be given by the argument of the amplitude $a_{R \ell}$ :

$$
\delta_{R \ell}=\arg a_{R \ell} .
$$

Using the asymptotics of Riccati-Bessel functions as $k R \gg \ell(\ell+1)$ and the asymptotics of the regular Coulomb function as $k R \gg \ell(\ell+1)+\eta^{2}$, one obtains the asymptotics of $a_{R \ell}$ :

$$
a_{R \ell} \sim \mathrm{e}^{\mathrm{i}\left(\sigma_{\ell}-\eta \log 2 k R\right)} .
$$

Therefore, the asymptotics of the phase shift $\delta_{R \ell}$ when $k R \gg \ell(\ell+1)+\eta^{2}$ reads [11]

$$
\delta_{R \ell} \sim \sigma_{\ell}-\eta \log 2 k R .
$$

The above procedure describes how the partial waves $v_{\ell}(r, k)$ can be constructed. Then the wavefunction $v(r, u, k)$ is given by the infinite sum over momenta $\ell$ :

$$
v(r, u, k)=\frac{1}{k r} \sum_{\ell=0}^{\infty}(2 \ell+1) \mathrm{i}^{\ell} v_{\ell}(r, k) P_{\ell}(u) .
$$

This function satisfies the three-dimensional Schrödinger equation (1) with the potential $V_{R}$ taken instead of $V_{\mathrm{c}}$.

Before proceeding further, we would like to point out that the convergence of the partial wave series for the scattering solutions should be considered with care, especially for the 
case of long-range potentials [12]. The most reliable method is by using distribution theory. Consider an infinitely differentiable test function $f(u) \in C^{\infty}(-1,1)$. By multiplying both sides of (17) with $f(u)$ and integrating over $u$, we obtain

$$
\int_{-1}^{1} \mathrm{~d} u v(r, u, k) f(u)=\frac{1}{k r} \sum_{\ell=0}^{\infty}(2 \ell+1) \mathrm{i}^{\ell} v_{\ell}(r, k) \int_{-1}^{1} \mathrm{~d} u P_{\ell}(u) f(u) .
$$

Introducing the Fourier coefficients with respect to the Legendre polynomials

$$
f_{\ell}=(2 \ell+1) \int_{-1}^{1} \mathrm{~d} u P_{\ell}(u) f(u)
$$

equation (18) can be rewritten in the following form:

$$
\int_{-1}^{1} \mathrm{~d} u v(r, u, k) f(u)=\frac{1}{k r} \sum_{\ell=0}^{\infty} \mathrm{i}^{\ell} v_{\ell}(r, k) f_{\ell} .
$$

The series on the right-hand side is absolutely and uniformly convergent in the interval $0<r<\infty$ since the set of Fourier coefficients $f_{\ell}$ itself forms the absolutely convergent series, and the terms of the series (20) can be estimated as

$$
\left|v_{\ell}(r, k) f_{\ell}\right| \leqslant C\left|f_{\ell}\right|,
$$

where $C$ is some constant. Thus, the leading term of the asymptotics of the series when $r \rightarrow \infty$ is now determined by the asymptotics of a certain number of coefficients $v_{\ell}(r, k)$, whilst the tail of the series is negligible. The detailed description of using such an approach for the partial wave series summation can be found in [12]. In the following discussion, we treat the partial series in the sense described above while assuming formulae such as (18), (20) implicitly. We also extend this technique to the operators acting on the square integrable functions of the angular variable $u$.

\subsection{Properties of the solution for $r \leqslant R$}

For $r \leqslant R$, equation (17) yields

$$
v(r, u, k)=\frac{1}{k r} \sum_{\ell=0}^{\infty}(2 \ell+1) \mathrm{i}^{\ell} a_{R \ell} F_{\ell}(\eta, k r) P_{\ell}(u) .
$$

The right-hand side of (22) is the series in Legendre polynomials [10]. The polynomials $P_{\ell}$ form an orthogonal and complete set of functions on the interval $(-1,1)$ with respect to the scalar product

$$
\langle f \mid g\rangle=\int_{-1}^{1} \mathrm{~d} u f^{*}(u) g(u)
$$

where the asterisk indicates the complex conjugate. The orthogonality and completeness conditions for $P_{\ell}$ are

$$
\begin{aligned}
& \int_{-1}^{1} \mathrm{~d} u P_{\ell}(u) P_{\lambda}(u)=\frac{2}{2 \ell+1} \delta_{\ell \lambda}, \\
& \sum_{\ell=0}^{\infty} \frac{2 \ell+1}{2} P_{\ell}(u) P_{\ell}\left(u^{\prime}\right)=\delta\left(u-u^{\prime}\right) .
\end{aligned}
$$

This set provides a basis for the $\mathcal{L}=L_{2}(-1,1)$ space of square integrable functions on the interval $(-1,1)$, with $(23)$ as the inner product and with $\|f\|=\langle f \mid f\rangle^{1 / 2}$ as the norm. In the 
following derivation, the elements of $\mathcal{L}$ will be denoted as vectors, e.g. $|f\rangle$. With this notation, (24) and (25) take the abbreviated form

$$
\begin{aligned}
& \left\langle P_{\ell} \mid P_{\lambda}\right\rangle=\frac{2}{2 \ell+1} \delta_{\ell \lambda}, \\
& \sum_{\ell=0}^{\infty} \frac{2 \ell+1}{2}\left|P_{\ell}\right\rangle\left\langle P_{\ell}\right|=\mathbf{I} .
\end{aligned}
$$

Here I denotes the unit operator in $\mathcal{L}$. Equation (22) now reads

$$
|v(r, k)\rangle=\frac{1}{k r} \sum_{\ell=0}^{\infty}(2 \ell+1) \mathrm{i}^{\ell} a_{R \ell} F_{\ell}(\eta, k r)\left|P_{\ell}\right\rangle .
$$

Here $|v(r, k)\rangle \in \mathcal{L}$ represents $v(r, u, k)$ as a function of $u$. From the form of (28), this can be recast into

$|v(r, k)\rangle=\sum_{\ell=0}^{\infty} a_{R \ell} \mathrm{e}^{-\mathrm{i} \sigma_{\ell}} \frac{2 \ell+1}{2}\left|P_{\ell}\right\rangle\left\langle P_{\ell}\left|\frac{1}{k r} \sum_{\lambda=0}^{\infty}(2 \lambda+1) \mathrm{i}^{\lambda} \mathrm{e}^{\mathrm{i} \sigma_{\lambda}} F_{\lambda}(\eta, k r)\right| P_{\lambda}\right\rangle$.

By comparing this equation with (3), one identifies the right-hand side of (29) with the action of the operator

$$
\mathbf{a}_{R}=\sum_{\ell=0}^{\infty} a_{R \ell} \mathrm{e}^{-\mathrm{i} \sigma_{\ell}} \frac{2 \ell+1}{2}\left|P_{\ell}\right\rangle\left\langle P_{\ell}\right|
$$

on the Coulomb wavefunction, which in $\mathcal{L}$ is represented by the vector

$$
\left|\psi_{\mathrm{c}}(r, k)\right\rangle=\frac{1}{k r} \sum_{\lambda=0}^{\infty}(2 \lambda+1) \mathrm{i}^{\lambda} \mathrm{e}^{\mathrm{i} \sigma_{\lambda}} F_{\lambda}(\eta, k r)\left|P_{\lambda}\right\rangle .
$$

Thus, we have obtained the central focus of this part of the derivation, which establishes the relation between the solution of the Schrödinger equation with the sharply cut-off potential $V_{R}$ and the Coulomb wavefunction for $r \leqslant R$. It has the form

$$
|v(r, k)\rangle=\mathbf{a}_{R}\left|\psi_{\mathrm{c}}(r, k)\right\rangle \text {. }
$$

The inverse identity also holds true, yielding

$$
\left|\psi_{\mathrm{c}}(r, k)\right\rangle=\mathbf{a}_{R}^{-1}|v(r, k)\rangle \text {. }
$$

The exact form of $\mathbf{a}_{R}^{-1}$ is easily calculated from the orthogonality and completeness of Legendere polynomials

$$
\mathbf{a}_{R}^{-1}=\sum_{\ell=0}^{\infty} a_{R \ell}^{-1} \mathrm{e}^{\mathrm{i} \sigma_{\ell}} \frac{2 \ell+1}{2}\left|P_{\ell}\right\rangle\left\langle P_{\ell}\right|
$$

Representation (11) guarantees $\left|a_{R, \ell}\right| \neq 0$, and consequently the inverse is correctly defined by (34). Formulae (32), (33) are the main results of this section, and they provide us with the representations of the Coulomb wavefunction in terms of the wavefunction $v(r, u, k)$. These representations are valid for an arbitrary value of the screening radius $R$.

A subsequent reduction of the complexity of the representations (32) and (33) can be observed if $R \rightarrow \infty$ by studying the asymptotics of the operator $\mathbf{a}_{R}$. The operator $\mathbf{a}_{R}$ can be simplified as $R \rightarrow \infty$ with the help of the asymptotics (15) of the coefficients $a_{R \ell}$. The natural topology for calculating the asymptotics of $\mathbf{a}_{R}$ is the strong operator topology in $\mathcal{L}$, i.e. when the asymptotics of vectors $\mathbf{a}_{R}|f\rangle$ in $\mathcal{L}$ is considered as $R \rightarrow \infty$. Construct a vector 
$\left|g_{R}\right\rangle=\mathbf{a}_{R}|f\rangle$ with an arbitrary $|f\rangle \in \mathcal{L}$ and then represent this vector as a sum of two terms $\left|g_{R}\right\rangle=\left|g_{L R}\right\rangle+\left|g_{R}^{L}\right\rangle$, where

$$
\begin{aligned}
& \left|g_{L R}\right\rangle=\sum_{\ell=0}^{L} a_{R \ell} \mathrm{e}^{-\mathrm{i} \sigma_{\ell}} \frac{2 \ell+1}{2}\left|P_{\ell}\right\rangle\left\langle P_{\ell} \mid f\right\rangle, \\
& \left|g_{R}^{L}\right\rangle=\sum_{\ell=L+1}^{\infty} a_{R \ell} \mathrm{e}^{-\mathrm{i} \sigma_{\ell}} \frac{2 \ell+1}{2}\left|P_{\ell}\right\rangle\left\langle P_{\ell} \mid f\right\rangle .
\end{aligned}
$$

The second sum can be made arbitrarily small by the choice of $L$. Indeed, due to the known properties of the Riccati-Hankel function and of the regular Coulomb function [10] it is seen that the coefficients (11) are bounded $\left|a_{R \ell}\right| \leqslant C$, then

$$
\left\|g_{R}^{L}\right\|^{2} \leqslant C^{2} \sum_{\ell=L+1}^{\infty} \frac{(2 \ell+1)}{2}\left|\left\langle P_{\ell} \mid f\right\rangle\right|^{2} .
$$

The Parseval identity for the series in Legendre polynomials

$$
\sum_{\ell=0}^{\infty} \frac{(2 \ell+1)}{2}\left|\left\langle P_{\ell} \mid f\right\rangle\right|^{2}=\|f\|^{2},
$$

shows that the series on the left-hand side of (37) is convergent and, as a consequence, its tail

$$
\sum_{\ell=L+1}^{\infty} \frac{(2 \ell+1)}{2}\left|\left\langle P_{\ell} \mid f\right\rangle\right|^{2}
$$

can be made arbitrarily small if $L$ is taken to be sufficiently large. From this we conclude that the infinite sum on the right-hand side of the inequality (36) can be made arbitrarily small if $L$ is large enough. Thus, for any small $\epsilon>0$, there exists an integer $L_{0}>0$ such that for all $L \geqslant L_{0}$ the inequality holds

$$
\left\|g_{R}^{L}\right\|^{2} \leqslant C^{2} \epsilon
$$

Consider now the vector $\left|g_{L_{0} R}\right\rangle$. Since $L_{0}$ is finite there always exists a value of $R$ such that the condition $k R \gg L_{0}\left(L_{0}+1\right)+\eta^{2}$ is fulfilled. More precisely, the asymptotics of the RiccatiHankel function and of the regular Coulomb function can be used for evaluating Wronskians in (11), as indicated in (15), to arrive at the inequality

$$
\left|a_{R L_{0}}-\mathrm{e}^{\mathrm{i}\left(\sigma_{L_{0}}-\eta \log 2 k R\right)}\right| \leqslant \epsilon^{1 / 2} .
$$

Similar inequalities where $L_{0}$ is replaced by $\ell$ for all $\ell \leqslant L_{0}$ are obviously also true. With these inequalities, we obtain

$$
\|\left|g_{L_{0} R}\right\rangle-\mathrm{e}^{-\mathrm{i} \eta \log 2 k R} \mathbf{I}_{L_{0}}|f\rangle\left\|^{2} \leqslant \epsilon\right\| f \|^{2},
$$

where

$$
\mathbf{I}_{L_{0}}=\sum_{\ell=0}^{L_{0}} \frac{2 \ell+1}{2}\left|P_{\ell}\right\rangle\left\langle P_{\ell}\right| .
$$

Combining inequalities (39) and (41) with the definition of $\left|g_{R}\right\rangle$, we obtain the final estimate

$$
\|\left(\mathbf{a}_{R}-\mathrm{e}^{-\mathrm{i} \eta \log 2 k R} \mathbf{I}_{L_{0}}\right)|f\rangle \|^{2} \leqslant \epsilon\left(C^{2}+\|f\|^{2}\right) .
$$

With this estimate, $L_{0}$ can be extended up to infinity and the inequality with $\mathbf{I}$ instead of $\mathbf{I}_{L_{0}}$ is also valid. Thus, the final result for the asymptotics of the operator $\mathbf{a}_{R}$ is formulated as follows. 
Theorem 1. For any arbitrarily small $\epsilon>0$ there exists $R$ such that the representation

$$
\mathbf{a}_{R}=\mathrm{e}^{-\mathrm{i} \eta \log 2 k R} \mathbf{I}+\mathbf{O}(\epsilon)
$$

holds true. Here the norm of a residual operator $\mathbf{O}(\epsilon)$ acting on any vector in $\mathcal{L}$ is of the order $\epsilon$ as in (43).

On applying theorem 1 to the vector $|f\rangle=\left|\psi_{\mathrm{c}}(r, k)\right\rangle$, the asymptotic form of relation (32) is obtained:

$$
|v(r, k)\rangle=\mathrm{e}^{-\mathrm{i} \eta \log 2 k R}\left|\psi_{\mathrm{c}}(r, k)\right\rangle+\mathbf{O}(\epsilon)\left|\psi_{\mathrm{c}}(r, k)\right\rangle .
$$

The alternative is given by

$$
\left|\psi_{\mathrm{c}}(r, k)\right\rangle=\mathrm{e}^{\mathrm{i} \eta \log 2 k R}\left[|v(r, k)\rangle-\mathbf{O}(\epsilon)\left|\psi_{\mathrm{c}}(r, k)\right\rangle\right] .
$$

These two last formulae provide the strict basis for the problem of the asymptotic regularization $[11,16]$, which is needed when constructing the three-dimensional Coulomb wavefunction from the solution of the Schrödinger equation with the screened Coulomb potential $V_{R}$.

Although the asymptotic regularizing factor $\mathrm{e}^{\mathrm{i} \eta \log 2 k R}$ is numerical and does not depend on the angular variable $u$, the general factor $\mathbf{a}_{R}$ is the operator in the angular space $\mathcal{L}$. This operator connects the two solutions $v(r, u, k)$ and $\psi_{\mathrm{c}}(r, u, k)$ to the same equations for $r \leqslant R$. This does not lead to a contradiction since the operator $\mathbf{a}_{R}$, or more precisely its extension on the three-dimensional space for which we will keep the same notation $\mathbf{a}_{R}$, commutes with the Hamiltonian $\mathbf{H}_{\mathrm{c}}=-\Delta_{\mathbf{r}}+V_{\mathrm{c}}(r)$ :

$$
\mathbf{a}_{R} \mathbf{H}_{\mathrm{c}}=\mathbf{H}_{\mathrm{c}} \mathbf{a}_{R} \text {. }
$$

Thus, if the function $v(r, u, k)$ obeys the Schrödinger equation for $r \leqslant R$

$$
\left(\mathbf{H}_{\mathrm{c}}-k^{2}\right) v(r, u, k)=0,
$$

then one obtains

$$
\begin{aligned}
\left(\mathbf{H}_{\mathrm{c}}-k^{2}\right) v(r, u, k) & =\left(\mathbf{H}_{\mathrm{c}}-k^{2}\right) \mathbf{a}_{R} \psi_{\mathrm{c}}(r, u, k) \\
& =\mathbf{a}_{R}\left(\mathbf{H}_{\mathrm{c}}-k^{2}\right) \psi_{\mathrm{c}}(r, u, k)=0 .
\end{aligned}
$$

For the sake of completeness, it is worth giving the explicit representation of the operator $\mathbf{a}_{R}$ acting on the functions of the angular variable:

$$
\mathbf{a}_{R} f(r, u, k)=\int_{-1}^{1} \mathrm{~d} u^{\prime} a_{R}\left(u, u^{\prime}\right) f\left(r, u^{\prime}, k\right) .
$$

Here the kernel $a_{R}\left(u, u^{\prime}\right)$ in accordance with (30) is represented by

$$
a_{R}\left(u, u^{\prime}\right)=\sum_{\ell=0}^{\infty} a_{R \ell} \mathrm{e}^{-\mathrm{i} \sigma_{\ell}} \frac{2 \ell+1}{2} P_{\ell}(u) P_{\ell}\left(u^{\prime}\right) .
$$

This kernel is identical to the function (5) from [17] after respective unification of notations.

\subsection{Properties of the solution for $r \geqslant R$}

For $r \geqslant R$, the function $v_{\ell}(r, k)$ has the form (10). The summation over $\ell$ leads to the three-dimensional solution

$$
v(r, u, k)=\mathrm{e}^{\mathrm{i} r k u}+v_{\mathrm{sc}}(r, u, k),
$$

where the scattered part has the form

$$
v_{\mathrm{sc}}(r, u, k)=\frac{1}{k r} \sum_{\ell=0}^{\infty}(2 \ell+1) \mathrm{i}^{\ell} A_{R \ell} \hat{h}_{\ell}^{+}(k r) P_{\ell}(u) .
$$


Using standard arguments, one arrives at the asymptotics of $v_{\mathrm{sc}}$ as $k r \rightarrow \infty$ :

$$
v_{\mathrm{sc}}(r, u, k) \sim A_{R}(u, k) \mathrm{e}^{\mathrm{i} k r} / r
$$

with the partial wave representation for the scattering amplitude $A_{R}(u, k)$ given by

$$
A_{R}(u, k)=\frac{1}{k} \sum_{\ell=0}^{\infty}(2 \ell+1) A_{R \ell} P_{\ell}(u) .
$$

If $R \rightarrow \infty$, the amplitude $A_{R}(u, k)$ can be represented through the Coulomb scattering amplitude. In order to demonstrate this, one needs to use the representation of the amplitude through the phase shift (13) and its asymptotics (16). The following representation is first derived:

$$
A_{R}(u, k) \sim \frac{1}{k} \sum_{\ell=0}^{\infty}(2 \ell+1) \frac{\mathrm{e}^{\mathrm{i} 2\left(\sigma_{\ell}-\eta \log 2 k R\right)}-1}{2 \mathrm{i}} P_{\ell}(u) .
$$

By using the standard partial wave expansion of the Coulomb scattering amplitude $A_{\mathrm{c}}$,

$$
A_{\mathrm{c}}(u, k)=\frac{1}{k} \sum_{\ell=0}^{\infty}(2 \ell+1) \frac{\mathrm{e}^{\mathrm{i} 2 \sigma_{\ell}}-1}{2 \mathrm{i}} P_{\ell}(u),
$$

representation (56) can be transformed to the form

$A_{R}(u, k) \sim \mathrm{e}^{-\mathrm{i} 2 \eta \log 2 k R} A_{\mathrm{c}}(u, k)-\frac{2}{k} \mathrm{e}^{-\mathrm{i} \eta \log 2 k R} \sin (\eta \log 2 k R) \sum_{\ell=0}^{\infty} \frac{2 \ell+1}{2} P_{\ell}(u)$.

The sum in the second term of (58) can easily be evaluated with the help of the completeness of the Legendre polynomials (25) and by taking into account that $P_{\ell}(1)=1$ :

$$
\sum_{\ell=0}^{\infty} \frac{2 \ell+1}{2} P_{\ell}(u)=\delta(1-u)
$$

Here the delta function is understood as in [12]:

$$
\int_{-1}^{1} \mathrm{~d} u \delta(1-u) f(u)=f(1) .
$$

Introducing (59) into formula (58), we obtain the final form of the asymptotics of the amplitude (55):

$$
A_{R}(u, k) \sim \mathrm{e}^{-2 \mathrm{i} \eta \log 2 k R} A_{\mathrm{c}}(u, k)-\frac{2}{k} \mathrm{e}^{-\mathrm{i} \eta \log 2 k R} \sin (\eta \log 2 k R) \delta(u-1) .
$$

This is one of the main results of this section. One can recognize in the first term of (61) the regularization factor, which was derived in [12]. The second term with the strong delta function singularity was not known until now and, as demonstrated in the analysis shown above, its exact form can be obtained by the accurate summation of all partial terms.

\section{The scattering problem for the potential $V^{R}$}

In this section, the approach detailed in paper [5] is followed to construct the solution to the partial wave equation

$$
\left(-\frac{\mathrm{d}^{2}}{\mathrm{~d} r^{2}}+\frac{\ell(\ell+1)}{r^{2}}+V^{R}(r)-k^{2}\right) w_{\ell}(r, k)=0
$$


for the potential $V^{R}$. The exact representation for $w_{\ell}(r, k)$ is of the form

$$
w_{\ell}(r, k)=a_{\ell}^{R} \hat{j}_{\ell}(k r)
$$

provided $r \leqslant R$. For $r \geqslant R$, this gives

$$
w_{\ell}(r, k)=\mathrm{e}^{\mathrm{i} \sigma_{\ell}} F_{\ell}(\eta, k r)+A_{\ell}^{R} u^{+}(\eta, k r) .
$$

Here $u^{+}(\eta, k r)=\mathrm{e}^{-\mathrm{i} \sigma_{\ell}}\left(G_{\ell}+\mathrm{i} F_{\ell}\right)$, and $G_{\ell}$ is the irregular Coulomb function [10]. Similar to (11) and (12), the parameters $a_{\ell}^{R}$ and $A_{\ell}^{R}$ should be found by matching representations (63) and (64) for the function $w_{\ell}(k r)$ and for its first derivative at the point $r=R$. This leads to the expressions

$$
\begin{aligned}
& a_{\ell}^{R}=\mathrm{e}^{\mathrm{i} \sigma_{\ell}} W_{R}\left(F_{\ell}, u_{\ell}^{+}\right) / W_{R}\left(\hat{j_{\ell}}, u_{\ell}^{+}\right), \\
& A_{\ell}^{R}=\mathrm{e}^{\mathrm{i} \sigma_{\ell}} W_{R}\left(F_{\ell}, \hat{j}_{\ell}\right) / W_{R}\left(\hat{j_{\ell}}, u_{\ell}^{+}\right) .
\end{aligned}
$$

The phase shift $\delta_{\ell}^{R}$ is introduced by the standard representation for the amplitude $A_{\ell}^{R}$ :

$$
A_{\ell}^{R}=\mathrm{e}^{\mathrm{i} 2 \sigma_{\ell}} \frac{\mathrm{e}^{\mathrm{i} 2 \delta_{\ell}^{R}}-1}{2 \mathrm{i}}
$$

For large values of $R$ such that $k R \gg \ell(\ell+1)+\eta^{2}$ the asymptotics of the regular Coulomb function and the Riccati-Bessel function can be used to obtain the following asymptotic representations for amplitudes (65) and (66):

$$
\begin{aligned}
& a_{\ell}^{R} \sim \mathrm{e}^{\mathrm{i} \eta \log 2 k R}, \\
& A_{\ell}^{R} \sim \mathrm{e}^{\mathrm{i} 2 \sigma_{\ell}} \frac{\mathrm{e}^{\mathrm{i} 2\left(\eta \log 2 k R-\sigma_{\ell}\right)}-1}{2 \mathrm{i}} .
\end{aligned}
$$

The solution to the three-dimensional Schrödinger equation is given by the sum over the momenta $\ell$ as

$$
w(r, u, k)=\frac{1}{k r} \sum_{\ell=0}^{\infty}(2 \ell+1) \mathrm{i}^{\ell} w_{\ell}^{R}(r, k) P_{\ell}(u) .
$$

As in the previous section, this function takes special forms on the intervals $0<r \leqslant R$ and $r \geqslant R$. The $\mathcal{L}$ vectors will be used as above for formulating results. For $0<r \leqslant R$, the vector $|w(r, k)\rangle$ can be represented in terms of the vector $\left|\psi_{0}(r, k)\right\rangle$, which represents the plane wave $\psi_{0}(r, u, k)=\mathrm{e}^{\mathrm{i} r k u}$ as follows:

$$
|w(r, k)\rangle=\mathbf{a}^{R}\left|\psi_{0}(r, k)\right\rangle .
$$

The operator $\mathbf{a}^{R}$ is represented by

$$
\mathbf{a}^{R}=\sum_{\ell=0}^{\infty} a_{\ell}^{R} \frac{2 \ell+1}{2}\left|P_{\ell}\right\rangle\left\langle P_{\ell}\right| .
$$

The asymptotics of this operator as $R \rightarrow \infty$ can be evaluated in the same way as in the previous section. The final result should again be understood in the sense of the strong operator topology in $\mathcal{L}$. It reads

$$
\mathbf{a}^{R} \sim \mathrm{e}^{\mathrm{i} \eta \log 2 k R} \mathbf{I} .
$$

This formula shows that, asymptotically, as $R \rightarrow \infty$,

$$
\mathbf{a}^{R} \simeq \mathbf{a}_{R}^{-1}
$$

10 
For $r \geqslant R$, the function $w(r, u, k)$ is given by the expression

$$
w(r, u, k)=\psi_{\mathrm{c}}(r, u, k)+w_{\mathrm{sc}}(r, u, k),
$$

where

$$
w_{\mathrm{sc}}(r, u, k)=\sum_{\ell=0}^{\infty}(2 \ell+1) \mathrm{i}^{\ell} A_{\ell}^{R} u_{\ell}^{+}(\eta, k r) P_{\ell}(u) .
$$

Using standard arguments, we obtain the asymptotics of $w_{\mathrm{sc}}(r, u, k)$ as $k r \rightarrow \infty$ :

$$
w_{\text {sc }}(r, u, k) \sim A^{R}(u, k) \mathrm{e}^{\mathrm{i}(k r-\eta \log 2 k r)} / r .
$$

Here the partial wave decomposition for the amplitude is expressed as

$$
A^{R}(u, k)=\frac{1}{k} \sum_{\ell=0}^{\infty}(2 \ell+1) A_{\ell}^{R} P_{\ell}(u) .
$$

If $R \rightarrow \infty$, the asymptotics of the amplitude $A^{R}(u, k)$ should be combined with the Coulomb amplitude $A_{\mathrm{c}}(u, k)$ in order to form the total amplitude of the outgoing spherical wave for the function (75). In this case, we obtain

$$
A_{\mathrm{c}}(u, k)+A^{R}(u, k) \sim \frac{2}{k} \mathrm{e}^{\mathrm{i} \eta \log 2 k R} \sin (\eta \log 2 k R) \delta(1-u) .
$$

Comparing with the representation (61) for the amplitude $A_{R}(u, k)$, one finds the relation

$$
A^{R} \simeq-\mathrm{e}^{2 \mathrm{i} \eta \log 2 k R} A_{R},
$$

which holds for large values of $R$. This relation can be proved independently by comparing the partial wave series for the amplitudes $A^{R}(u, k)$ and $A_{R}(u, k)$.

The opposite limit as $R \rightarrow 0$ has a certain interest for the case of the potential $V^{R}$. It is obvious that $V^{R} \rightarrow V_{\mathrm{c}}$. A similar effect can be expected for the limit of the wavefunction $w(r, u, k) \rightarrow \psi_{\mathrm{c}}(r, u, k)$. The proof is based on the following asymptotics for the coefficients $a_{\ell}^{R}$ and $A_{\ell}^{R}$ :

$$
\begin{aligned}
& a_{\ell}^{R} \sim \mathrm{e}^{\mathrm{i} \sigma_{\ell}} C_{\ell}(2 \ell+1) ! !, \\
& A_{\ell}^{R} \sim \mathrm{e}^{\mathrm{i} 2 \sigma_{\ell}} \frac{C_{\ell}^{2} \eta}{\ell+1}(k R)^{2 \ell+2} .
\end{aligned}
$$

Here $C_{\ell}$ is the standard Coulomb normalization factor [10]. It is easy to see that asymptotically as $R \rightarrow 0$

$$
a_{\ell}^{R} \hat{j}_{\ell}(k r) \sim \mathrm{e}^{\mathrm{i} \sigma_{\ell}} F_{\ell}(\eta, k r)
$$

for all $0<r \leqslant R$. When $R \rightarrow 0$, the function $u^{+}(\eta, k r)$ becomes singular as $r \rightarrow R$ :

$$
u^{+}(\eta, k r) \propto r^{-l} \text {. }
$$

At the same time, the amplitude $A_{\ell}^{R}$ behaves as

$$
A_{\ell}^{R} \propto R^{2(l+1)} .
$$

Hence, for all $R \leqslant r<\infty$, one obtains

$$
\max _{r \in[R, \infty)}\left|A_{\ell}^{R} u_{\ell}^{+}(\eta, k r)\right|=O\left(R^{\ell+2}\right),
$$

which shows that the term $A_{\ell}^{R} u_{\ell}^{+}(\eta, k r)$ vanishes faster than the leading term $\mathrm{e}^{\mathrm{i} \sigma_{\ell}} F_{\ell}(\eta, k r)=$ $O\left(r^{\ell+1}\right)$ when $r \rightarrow R$ and $R \rightarrow 0$. Using these estimates in (64), one readily arrives at the statement

$$
w(r, u, k) \sim \psi_{\mathrm{c}}(r, u, k)
$$

when $R \rightarrow 0$. 


\section{The driven Schrödinger equation for the Coulomb scattering problem}

In our recent study [5], we demonstrated that the inhomogeneous partial wave Schrödinger equation for the scattered part of the wavefunction with purely outgoing boundary conditions can be constructed and then successfully employed for solving the scattering problem for long-range potentials. The key element of this approach is the solution of the partial wave Schrödinger equation for the potential $V^{R}$. The three-dimensional approach can now be formulated with the help of the solution from the preceding section. The Hamiltonian $\mathbf{H}_{\mathrm{c}}$ is represented by

$$
\mathbf{H}_{\mathrm{c}}=-\Delta_{\mathbf{r}}+V_{R}(r)+V^{R}(r)
$$

and the wavefunction $\psi_{\mathrm{c}}$ as

$$
\psi_{\mathrm{c}}(r, u, k)=w(r, u, k)+\psi_{R}(r, u, k) .
$$

Here $w(r, u, k)$ is the wavefunction for the potential $V^{R}$ :

$$
\left(-\Delta_{\mathbf{r}}+V^{R}(r)-k^{2}\right) w(r, u, k)=0
$$

constructed in the preceding section. The function $\psi_{R}(r, u, k)$ obeys the inhomogeneous (driven) equation

$$
\left(-\Delta_{\mathbf{r}}+V_{\mathrm{c}}(r)-k^{2}\right) \psi_{R}(r, u, k)=-V_{R}(r) w(r, u, k)
$$

and the purely outgoing boundary conditions as $r \rightarrow \infty, r \geqslant R$

$$
\psi_{R}(r, u, k) \sim \mathcal{A}_{R}(u, k) \mathrm{e}^{\mathrm{i}(k r-\eta \log 2 k r)} / r .
$$

It is seen from the definition that the amplitude $\mathcal{A}_{R}$ is given in terms of $A^{R}$ through (78) by

$$
\mathcal{A}_{R}=-A^{R} \text {. }
$$

For $r \leqslant R$, it is useful to employ the interpretation of the functions as vectors in $\mathcal{L}$. In this notations, $\psi_{R}$ takes the form

$$
\left|\psi_{R}(r, k)\right\rangle=\left|\psi_{\mathrm{c}}(r, k)\right\rangle-\mathbf{a}^{R}\left|\psi_{0}(r, k)\right\rangle,
$$

and equation (91) becomes

$$
\left(\mathbf{H}_{\mathrm{c}}-k^{2}\right)\left|\psi_{R}(r, k)\right\rangle=-\mathbf{a}^{R} V_{R}\left|\psi_{0}(r, k)\right\rangle .
$$

Multiplying (94) by $\left(\mathbf{a}^{R}\right)^{-1}$ and using (33), one arrives at the representation

$$
\left(\mathbf{a}^{R}\right)^{-1}\left|\psi_{R}(r, k)\right\rangle=\left(\mathbf{a}_{R} \mathbf{a}^{R}\right)^{-1}|v(r, k)\rangle-\left|\psi_{0}(r, k)\right\rangle,
$$

which can be reduced using the asymptotic relation (74) for large values of $R$ to

$$
\left(\mathbf{a}^{R}\right)^{-1}\left|\psi_{R}(r, k)\right\rangle \simeq|v(r, k)\rangle-\left|\psi_{0}(r, k)\right\rangle .
$$

By its construction, the function

$$
\left|\phi_{R}(r, k)\right\rangle=\left(\mathbf{a}^{R}\right)^{-1}\left|\psi_{R}(r, k)\right\rangle
$$

obeys the equation

$$
\left(\mathbf{H}_{\mathrm{c}}-k^{2}\right)\left|\phi_{R}(r, k)\right\rangle=-V_{R}\left|\psi_{0}(r, k)\right\rangle .
$$

Following (96), the function $\phi_{R}(r, k)$ for $r \leqslant R$ and large values of $R$ can be represented as

$$
\left|\phi_{R}(r, k)\right\rangle \simeq|v(r, k)\rangle-\left|\psi_{0}(r, k)\right\rangle \text {. }
$$

Equation (98) is the desired three-dimensional driven equation, which can be used for solving the Coulomb scattering problem by the complex rotation method. The formulation of the scattering problem on the basis of equation (98) obeys two necessary conditions, which are needed for the application of the complex rotation method, i.e. (i) the solution $\phi_{R}(r, u, k)$ has the purely outgoing asymptotics 


$$
\phi_{R}(r, u, k) \sim\left(\mathbf{a}^{R}\right)^{-1} \mathcal{A}_{R} \mathrm{e}^{\mathrm{i}(k r-\eta \log 2 k r)} / r
$$

and (ii) the inhomogeneous term on the right-hand side of (98) vanishes outside of the sphere of the radius $R$.

\section{Conclusion}

New results on the structure of the solutions to the three-dimensional Schrödinger equation for the sharply cut-off Coulomb potential have been derived. For the potential $V_{R}$, which coincides with the Coulomb potential for all $r \leqslant R$, it was found that the wavefunction is proportional to the Coulomb wavefunction up to an operator factor. This operator acts as an integral operator over the spherical angular variable. The operator $\mathbf{a}_{R}$ is reduced to the multiplication by the constant $\mathrm{e}^{-\mathrm{i} \eta \log 2 k R}$ only asymptotically as $R \rightarrow \infty$. This result clarifies the domain of validity for unjustified assumptions about the proportionality factor which was taken as a constant in [11] and [16]. The asymptotic representation of the scattering amplitude for the potential $V_{R}$ in the case where $R \rightarrow \infty$ in addition to the standard term $\mathrm{e}^{-\mathrm{i} 2 \eta \log 2 k R} A_{\mathrm{c}}$ also contains the extra term (61). It has fast oscillations as $R \rightarrow \infty$ and a strong delta-functional singularity in the forward scattering direction. To the best of our knowledge, this formula has been derived here for the first time. The representation for the scattering amplitude obtained in the recent paper [16] was derived from the incorrect form of the wavefunction in the region $r \leqslant R$ [17] and cannot be considered as a contra-result. The complete solution for the scattering problem for the potential $V^{R}$ is given in this paper for the first time. Formula (80) supports the complementary character of the two potentials in the sense that $V^{R}=V_{\mathrm{c}}-V_{R}$ and should be considered as the fact of the self-consistency of our treatment. The three-dimensional formulation of the driven Schrödinger equation, given in section 4, opens the way for forthcoming applications in the three-body systems along the line given in [6].

\section{Acknowledgments}

SLY and EY are grateful to Stockholm University for travel and support made possible under the bilateral agreement between Stockholm University and St Petersburg State University. The work of SLY and EY was partly supported by the Russian Foundation for Basic Research under the grant 08-02-01115-a. This work was supported in part by grants from the Swedish National Research Council.

\section{References}

[1] Shakeshaft R 2009 Phys. Rev. A 80012708

[2] Nuttall J and Cohen H L 1969 Phys. Rev. 1881542

[3] Rescigno T N, Baertschy M, Byrum D and McCurdy C W 1997 Phys. Rev. A 554253

[4] McCurdy C W, Baertschy M and Rescigno T N 2004 J. Phys. B: At. Mol. Opt. Phys. 37 R137

[5] Volkov M, Elander N, Yarevsky E and Yakovlev S L 2009 Europhys. Lett. 8530001

[6] Elander N, Volkov M V, Larson A, Stenrup M, Mezei J Z, Yarevsky E and Yakovlev S 2009 Few-Body Syst. 45197

[7] Temple G 1928 Proc. R. Soc. A 121673

[8] Gordon W 1928 Z. Phys. 48180

[9] Messiah A 1958 Quantum Mechanics (New York: Wiley)

[10] Abramowitz M and Stegun I A (eds) 1986 Handbook of Mathematical Functions (New York: Dover)

[11] Ford W F 1964 Phys. Rev. 133 B1616

Ford W F 1966 J. Math. Phys. 7626 
[12] Taylor J R 1974 Nuovo Cimento B 23313

Semon M D and Taylor J R 1975 Nuovo Cimento A 2648

[13] Gorshkov V G 1961 Sov. Phys._JETP 131037

Gorshkov V G 1965 Sov. Phys._JETP 20234

[14] Alt E O, Sandhas W and Ziegelmann H 1978 Phys. Rev. C 171981

[15] Deltuva A, Fonseca A C and Sauer P U 2005 Phys. Rev. C 71054005

[16] Glöckle W, Golak J, Skibiński R and Witała H 2009 Phys. Rev. C 79044003

[17] Kouzakov K A, Popov Yu V and Shablov V L 2010 Phys. Rev. C 81019801

[18] Deltuva A, Fonseca A C and Sauer P U 2010 Phys. Rev. C 81019802 